\title{
Y a-t-il une solution de continuité entre théorie et pratique?
}

Is There a Discontinuity between Theory and Practice?

\section{Serge Agostinelli et Marielle Metge}

\section{(2) OpenEdition}

\section{Journals}

Édition électronique

URL : http://journals.openedition.org/edc/5106

DOI : 10.4000/edc.5106

ISSN : 2101-0366

Éditeur

Université Lille-3

Édition imprimée

Date de publication : 1 juin 2013

Pagination : 43-58

ISBN : 978-2-917562-09-3

ISSN : $1270-6841$

Référence électronique

Serge Agostinelli et Marielle Metge, "Y a-t-il une solution de continuité entre théorie et pratique ? », Études de communication [En ligne], 40 | 2013, mis en ligne le 01 juin 2013, consulté le 19 avril 2019 URL : http://journals.openedition.org/edc/5106 ; DOI : 10.4000/edc.5106

Ce document a été généré automatiquement le 19 avril 2019

(c) Tous droits réservés 


\title{
Y a-t-il une solution de continuité entre théorie et pratique?
}

\author{
Is There a Discontinuity between Theory and Practice?
}

Serge Agostinelli et Marielle Metge

1 L'objectif de cet article est de montrer que les modèles théoriques sont au cœur de l'action humaine et qu'ils ne peuvent pas être disqualifiés face à l'évidence des pratiques. Autant le dire dès le début, il n'y a pas de rupture, le mélange est homogène, il est le résultat de la solution de la théorie dans les pratiques. Dans un premier temps nous expliquons pourquoi nous ne pouvons pas dissocier le théorique du pratique puis, nous donnons un exemple pragmatique d'une recherche située qui fonde leur fertilisation croisée. Dans cette recherche, le premier auteur assure le pilotage. Il a défini les tâches, les méthodes et les livrables. Il a également conduit les réunions qui ont permis aux professionnels d'associer leurs actions quotidiennes à des modèles théoriques. Il a particulièrement traduit les différences académiques généralement admises entre les notions de connaissance, compétence, capacité. Le second auteur, avec les deux autres collègues citées plus loin, a participé aux entretiens, au recueil des données et aux analyses.

2 La nécessaire finalisation des recherches, particulièrement en SHS, pousse l'hégémonie de la recherche sociale empirique et il n'y a donc rien d'étonnant à ce que les chercheurs lui trouvent une filiation directe avec le paradigme actuel dominant: le constructivisme. La recherche-action ne relèverait donc pas directement d'une posture épistémologique constructiviste, mais plus d'une volonté de contrôle des moyens mis à disposition sur un terrain. Ceci est particulièrement notable dans les organisations où les innovations, le changement, les stratégies déjà introduites, devraient bénéficier, dans la mesure du possible, d'un appui empirique.

3 Pour expliciter notre contrôle des moyens mis à disposition sur un terrain d'expérience, nous présentons une recherche qui a pour finalité la création d'un réseau de femmes chefs d'entreprise sur la région marseillaise. Au-delà d'un réseau socio-professionnel relationnel, la recherche doit définir (catégoriser, critérier) les compétences et capacités 
susceptibles de tisser des liens forts et des liens faibles entre les individus. Ce maillage doit donner à terme, la cartographie des relations qui permettraient de savoir avec qui travailler en fonction de la nature des projets de chacune des femmes chefs d'entreprise.

\section{Position du problème}

4 C'est théorique, théoriquement, en théorie : ces expressions ont pris en français un sens péjoratif. On en use pour dénoncer un prétendu vide de la pensée et une ignorance du réel (Muglioni, 2008: 4). Certains professionnels, soit ceux qui ont une activité rémunératrice reconnue par le sens commun, pensent que l'on ne peut être docteur, qu'en médecine. Cet a priori pose que le chercheur serait un rêveur décroché de la réalité car il n'est pas soumis à résultat. D'ailleurs, il croit pouvoir tirer la connaissance de toute chose de son esprit, alors que pour «le commun des mortels", elle ne peut venir que des expériences du quotidien.

5 La recherche-action se positionne comme démarche sociale des chercheurs, susceptible de répondre à ces critiques avec un double objectif : transformer la réalité et produire des connaissances concernant ces transformations (Hugon et Seibel, 1988, 13). Elle serait alors une stratégie qui oriente les livrables et détermine le choix des instruments méthodologiques d'un travail empirique qui repose sur l'observation et le classement des phénomènes. La finalité de l'acte de recherche tend à déceler des invariants, des régularités et des constantes (Nedeljkovic, 1975, 75) qui définissent alors modèles et théories.

\section{Corpus théorique}

6 Les pratiques qui structurent les organisations sont l'indice d'une subjectivité des acteurs, qui se démarquerait de l'objectivité supposée des ingénieurs et des administrateurs. On passe d'une organisation fondée sur des structures formelles issues des premières approches dites classiques dont les méthodes mettaient en œuvre la modélisation et les enquêtes, à une organisation production de savoirs issue d'une analyse des parties prenantes dont les méthodes mettent en avant, l'analyse des discours et des interactions (Rouleau, 2007). L'organisation devient une construction sociale de micro dispositifs de pouvoir et/ou de résistances qui interroge la nature de ses paradigmes et les réalités subjectives, ainsi que les conditions de la production de connaissances pour l'entreprise (Landrieux-Kartochian, 2010).

7 C'est sur ce dernier point que nous discutons la fertilisation croisée. Celle-ci questionne la genèse ou la racontabilité des connaissances professionnelles. Ici : si l'organisation est caractérisée par la production de savoir-faire ; si ces savoirs sont issus d'une analyse des parties prenantes à partir d'une analyse des discours et des interactions; alors, il convient de croiser l'analyse des discours sur les connaissances professionnelles (le pratique) pour en déduire ou avoir une trace des interactions qui sont la mise en relation de systèmes de pensée (le théorique). En effet, par leurs natures sporadique et éphémère, les interactions contribuent à remettre en question les contours d'une orthodoxie objective de la genèse des connaissances. D'un point de vue théorique il est maintenant admis que les connaissances peuvent être déclaratives sur des faits ou procédurales ou sur des plans d'action (Anderson, 1983). Ces manières de faire font le lien entre la pensée 
et l'action, car elles sont finalisées et se caractérisent par: la mise en œuvre de compétences en vue de la réalisation d'un but (Leplat, 1991); l'explication de la performance observée qui décrit l'organisation des connaissances construites par l'action et pour l'action (Samurçay et Pastré parlaient de travail, 1995).

En d'autres termes, si nous voulons atteindre le double objectif de transformer la réalité et produire des connaissances concernant ces transformations, il convient d'envisager la recherche comme une succession de situations dans lesquelles nous avançons à plusieurs et en considérant les actions, les compétences et les connaissances des partenaires non scientifiques comme autant de conditions supplémentaires de l'environnement de la recherche. Dès lors, la réification des actions et leur réduction sémantique (partielle ou globale) ne sont plus indispensables au bon fonctionnement de la recherche. D'un côté, le chercheur ne cherche plus à transformer un concept en un objet concret et de l'autre, le terrain ne regarde plus un concept comme s'il n'avait aucune relation avec les choses concrètes. Toute la difficulté de la recherche est alors de garantir un niveau de reconnaissance de chacun, car l'affaiblissement des points de vue théoriques en situation aboutit à un nivellement des concepts et la disparition de leur relation sur le terrain. Par exemple, dans une approche expérimentale, la disparition des relations entre concepts se reflète dans les réductions imposées aux variables et aux hypothèses pour leur manipulation. Tout ce qui n'est pas en rapport avec l'activité observée est écarté ou contrôlé et le chercheur peut ignorer des caractéristiques minorées. Avec une approche pragmatique, le chercheur rapproche les caractéristiques mineures du modèle théorique car il est difficile d'isoler les faits des caractéristiques de la situation sociale ou scientifique dans laquelle est conduite la recherche. Celle-ci est ici, une pratique située. En effet, lorsqu'il convient de définir une stratégie qui oriente la recherche et détermine le choix des instruments méthodologiques d'un travail empirique, le terme qui nous semble le plus adapté est celui de recherche-située. Nous faisons bien sûr référence ici, aux travaux de Suchman (1987). La compréhension mutuelle d'une recherche située peut être décrite par cinq propositions :

- Les protocoles de recherche sont construits en situation avec les partenaires;

- La négociation intervient lorsque l'activité généralement transparente devient problématique ;

- L'objectivité pratique des situations de recherche est construite plutôt que donnée ;

- La reconnaissance des postures réciproques est la ressource centrale pour la construction de l'objectivité des situations. Elle se construit généralement grâce à la communication et à une relation indicielle aux circonstances qu'elle suppose ;

- L'intelligibilité mutuelle est une conséquence des interactions. Elle est le résultat d'un compromis entre la nature indexicale de la communication et le nécessaire recours aux détails de la situation. Elle n'est pas décrite a priori par un corpus stable de significations partagées.

9 En fait le chercheur « doit réapprendre à voir le monde autour de lui ; dans certaines situations familières, il doit apprendre à voir de nouvelles formes. Le monde de ses recherches lui paraîtra ensuite, sur certains points, incommensurable avec celui dans lequel il habitait la veille» (Kuhn, $1972: 158)$. 


\section{Méthodologie}

10 Notre méthodologie pour une continuité entre théorie et pratique se fonde sur une recherche située que nous conduisons avec des Chefs d'Entreprise Féminins (ChEF) de la CGPEM13 ${ }^{1}$. Cette recherche répond à un double objectif. Le premier est stratégique, il est issu des échanges entre un professeur d'université et une femme chef d'entreprise, lors des rencontres Universités Entreprises ${ }^{2}$. La chef d'entreprise étant fortement impliquée dans les doctoriales d'Aix-Marseille Université, son souhait est d'impliquer davantage ses collègues dirigeantes d'entreprise dans une recherche académique. Le second est plus opérationnel, il demande la clarification de compétences spécifiques aux «valeurs féminines » pour la construction d'un réseau professionnel. Cette recherche s'est construite à partir d'une approche empirico-inductive qualitative critique, en privilégiant les entretiens et une analyse compréhensive des discours sur les valeurs qu'elles associent aux compétences et capacités.

\section{Contexte}

11 La demande vient donc du terrain et paradoxalement, elle est théorique sous la forme d'une information scientifique. Le but était d'apporter une clarification sur les différences que font les universitaires entre connaissances, compétences et capacités et d'en proposer une articulation. Cette information a été pour nous un exercice de traduction (au sens de Callon et Latour, 2006) alors que pour les ChEF, elle a été une forme de négociation entre les différents points de vue : une pratique normale de terrain. Elle a été en fait, significative d'une mise en commun de concepts: une pratique normale de recherche.

\section{Méthode}

12 L'aspect qualitatif de cette approche se traduit par l'intérêt attribué aux contextes du monde économique en PACA dans lesquels évoluent les ChEF. Opter pour une recherche empirico-inductive revient donc à accorder sa priorité aux pratiques et à ne pas formuler d'hypothèse a priori. En effet les acteurs de terrain cherchent généralement dans une équipe de chercheurs, un accompagnement à leur réflexion en temps réel (Huberman et Miles, 2003). Dès lors, notre recherche a pris une forme de réflexivité pour nous positionner dans une approche de questionnement et d'explicitation. Nous avons développé une analyse compréhensive des entretiens fondée sur l'observation pseudoparticipante. Pseudo, car, nous ne pouvons pas dire que nous avons une véritable immersion de longue durée sur le terrain. Elle est donc plutôt participative et occasionnelle mais, nous a permis d'avoir une compréhension du contexte socioéconomique-temporel propre à l'évolution de leur questionnement. Nous avons donc accordé une place importante aux notes prises à l'occasion des réunions et à la transcription directe des discours. L'observation n'est pas, à proprement parler, une observation des activités quotidiennes, mais une verbalisation proposée par les ChEF.

13 Le discours sur les pratiques est bien à l'articulation entre le faire et le penser, car il est l'expression d'un contexte d'action tout en participant en même temps à l'élaboration de ce contexte: décrire une situation c'est la constituer (Coulon, 1993). C'est aussi 
présupposer que les activités, par lesquelles les hommes produisent et gèrent les situations de leur vie organisée de tous les jours, sont identiques aux procédures utilisées pour rendre ces situations descriptibles (Garfinkel, 1967). En ce sens, les discours sont des formes opératoires révélatrices de l'action qu'il s'agit de rassembler et d'exploiter. Par exemple à la question: Quelles compétences êtes-vous disposées à partager avec le groupe ? Avec comme consigne : En face de chacune des compétences, décrire ce que cela permet de faire; la réponse a été: «Mes compétences relationnelles et ma facilité à parler ou présenter des idées. Je sais animer un groupe, je sais faire passer des idées ». La ChEF qui avance des compétences relationnelles avance aussi celles-ci, comme étant importantes pour le groupe. Nos observations, pendant les entretiens collectifs, corroborent cette affirmation. Cette personne prend facilement la parole et oriente le groupe. Elle dit donc ce qu'elle fait et le lien entre la pensée et l'action est bien effectif.

Dès lors, les premiers entretiens collectifs nous ont permis de constituer une base d'analyse pour le logiciel Tropes ${ }^{3}$. Tropes fait apparaître l'ossature d'un discours fondé sur l'analyse cognitivo-discursive (ACD - Ghiglione, Kekenbosch et Landré, 1995) et l'analyse propositionnelle du discours (ADP - Ghiglione et Blanchet, 1991). L'unité de découpage est la proposition. Il repère donc les phrases clés correspondant aux idées qui contiennent l'essentiel du sens et "trace» les relations entre les mots ou les classes d'équivalents fréquemment reliées. Chaque analyse débute donc par le rangement des différenciateurs sémantiques de l'univers de référence (ici, les connaissances, les compétences, les capacités). Par exemple, dans les entretiens, le mot «concevoir » est souvent relié aux termes présenter dans le tableau 1.

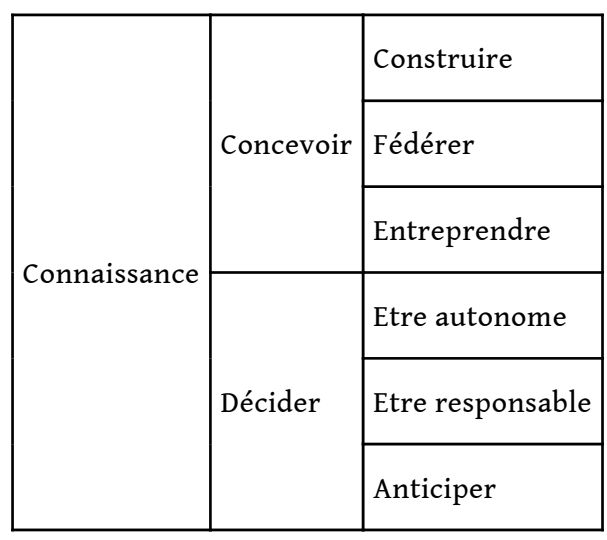

Tableau 1 : Termes associés à la supra catégorie [Connaissance]

Pour construire la classe d'équivalent de [connaissance] nous lui associons les verbes: construire, fédérer, entreprendre... autant de verbes qui donnent des variantes à la notion et qu'il faut organiser. Cette organisation est assurée par les scénarios qui enrichissent et filtrent les classes d'équivalents en fonction d'une stratégie d'analyse. Par exemple, si nous ajoutons un autre verbe (imaginer) en lien avec concevoir dans le groupe [connaissance]; le scénario lève l'ambigüité d'imaginer. Ce verbe sera toujours associé à [concevoir], quel que soit le discours analysé. Pour nous, il s'agit ici de considérer l'organisation des liens sémantiques entre les valeurs : une sorte d'état des lieux de leur système de valeurs et de pensée, appliqué à leurs activités. Cette base pose les variables clés de l'analyse structurelle des discours qu'elles auront à tenir sur leurs activités 
professionnelles et surtout ce qu'elles attendent d'un travail en commun, d'un réseau de partage de compétences spécifiques aux « valeurs féminines ».

La base d'analyse nous permet de disposer de la liste exhaustive des références utilisées par les femmes interrogées, références qui appartiennent aux catégories grammaticales suivantes : \{substantifs, verbes, adjectifs\} et aux catégories qui relèvent précisément de leurs valeurs : \{Concevoir; Organiser; Communiquer\}. Ces trois variables ne sont ici qu'à titre d'exemple, nous disposons pour l'analyse complète de: cinq supra catégories; dix super catégories; trente catégories sémantiques positives. L'ensemble est à doubler pour avoir les valeurs contrastées, car il s'agit de faire varier des jeux de scénarios d'analyses contrastées et donc de considérer pour chaque variable, l'item et son antonyme \{Relation; Individualisme\}.

\begin{tabular}{|c|c|c|c|c|c|}
\hline $\begin{array}{l}\text { Supra- } \\
\text { Catégorie }\end{array}$ & $\begin{array}{l}\text { Super- } \\
\text { Catégorie }\end{array}$ & $\begin{array}{l}\text { Catégorie } \\
\text { Sémantique Positif }\end{array}$ & $\begin{array}{l}\text { Catégorie } \\
\text { Sémantique } \\
\text { Négatif }\end{array}$ & $\begin{array}{l}\text { Super- } \\
\text { Catégorie }\end{array}$ & $\begin{array}{l}\text { Supra- } \\
\text { Catégorie }\end{array}$ \\
\hline \multirow{6}{*}{ Relation } & \multirow{3}{*}{$\begin{array}{l}\text { Communi- } \\
\text { cation }\end{array}$} & Dialoguer & Diffuser & \multirow{3}{*}{ Information } & \multirow{6}{*}{$\begin{array}{l}\text { Individua- } \\
\text { lisme }\end{array}$} \\
\hline & & Ecouter & Entendre & & \\
\hline & & Animer & Brider & & \\
\hline & \multirow{3}{*}{ Liaison } & Autre & Moi & \multirow{3}{*}{ Séparation } & \\
\hline & & Adapter & Dissocier & & \\
\hline & & Partager & Diviser & & \\
\hline
\end{tabular}

Tableau 2 : Exemple de hiérarchie catégorielle

17 Chaque catégorie sémantique est ensuite constituée des substantifs, verbes, adjectifs au sens propre ou figuré. Ainsi la catégorie «dialoguer» peut regrouper : bavarder (sens propre) et accoucher (sens figuré). Au final nous disposons d'un lexique de 1518 référents. A partir de ce lexique, nous élaborons le scénario qui permet d'identifier, de catégoriser et de comptabiliser automatiquement le lexique des compétences contenues dans les discours sur les pratiques, produits à l'oral ou à l'écrit. La seconde phase de la recherche consiste à passer des entretiens individuels et collectifs pour recueillir les discours sur : comment deux ChEF se reconnaissent mutuellement, l'une et l'autre; comment sont identifiées les compétences des collaborateurs...

In fine notre livrable s'articule autour de trois points: (a) une analyse des membres du groupe en termes de liens forts et faibles; quels sont les profils les plus actifs ou passifs; les moteurs et les suiveurs... (b) une analyse des capacités mises à la disposition de tous "Je peux... pour vous...»; (c) une analyse des performances les plus actives, les plus régulières... 


\section{Résultats}

19 En regard de l'objectif de cet article, il va de soi que nous avons présenté notre dispositif de recherche comme un exemple à nos propos et non ses résultats (cf. Arvanitakis et al., à paraître). L'intérêt de parler du terrain est bien ici d'expliquer comment s'opère la fertilisation croisée entre la recherche et l'action, entre le théorique et la pratique.

\section{La première fertilisation croisée est celle des compétences}

La recherche est amenée à tenir compte des finalités stratégiques des ChEF pour assurer une information pragmatique permettant de mettre les membres du groupe au même niveau d'analyse des projets envisagés. Les moyens accordés à notre recherche: disponibilité, participation... permettent aux ChEF de se mettre à distance de leur quotidien et d'accéder à une méthodologie scientifique d'analyse de leurs pratiques. Ces ChEF savent ce qu'elles font au quotidien mais n'arrivent pas à généraliser, car elles donnent des noms différents à leurs actions. Elles n'ont pas de langage commun autre que celui du métier ou du référentiel de compétences et ne mettent pas les mêmes " choses » sous les mêmes mots dès qu'il s'agit de phénomènes plus difficilement formalisables tels que les compétences, les capacités, les relations entre les valeurs...

21 Il ressort de cette première fertilisation : pour nous, une première catégorisation qui nous servira dans le montage des scénarios d'analyse des discours sur la finalité à donner au réseau de ChEF ; pour les ChEF, une hiérarchie de valeurs incontournables et communes. Cette hiérarchie laisse clairement apparaître que la compétence $n^{\circ} 1$ est la « conception ». Les ChEF sont capables de concevoir, leur première valeur fait donc référence au professionnel. Les deux suivantes font référence à l'autre et à soi, elles placent donc très rapidement des valeurs d'identité personnelle et de rapport au groupe. Les deux suivantes recentrent sur le professionnel puis encore les deux suivantes sur leur relation au groupe. Pour nos catégories nous obtenons donc: \{Concevoir; (Autre; Soi); (Organiser ; Connaître) ; (Communiquer ; Relation)\}.

\section{La deuxième fertilisation croisée est celle de la reconnaissance des pratiques}

Pour conduire cette recherche nous sommes sortis de notre laboratoire et nous agissons conjointement avec les participants dès le début de l'action. Nous partageons le but de transformer l'environnement professionnel à travers un processus d'investigation. Les points clés de l'analyse mettent en relief des construits théoriques liés à l'action des ChEF et chaque négociation est envisagée avec la perspective d'une construction intellectuelle qui permet de repérer les idées reçues ou celles qui sont systématiques et invisibles (Huberman et Miles, 2003). Ce processus réflexif, montre que le chercheur n'est pas un rêveur et qu'il est soumis à l'atteinte d'un résultat et montre également que le terrain a des préoccupations qui ne sont pas directement finalisables. En fait, nous mettons en place un réseau sémantique entre les concepts là où, les $\mathrm{ChEF}$ ne voyaient que de l'intuition féminine : liens sémantiques, «je sais » faire (compétence) et «je peux » faire (capacité). 


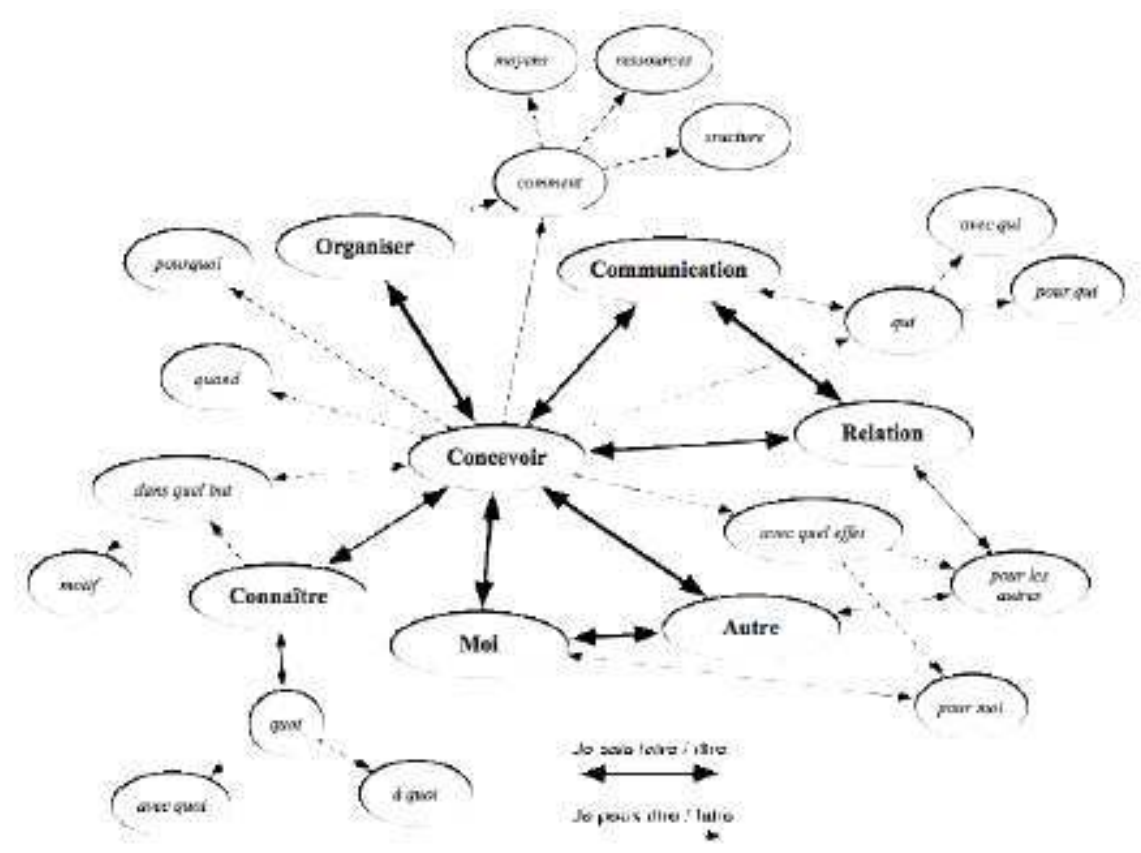

Figure 1 : Exemple de cartographie d'une corrélation entre les variables qui caractérisent les ChEF et leurs comportements. Cette stratégie aurait déterminé le choix des instruments différents. Ici, les données sont colligées et communiquées aux membres du groupe pour validation et mise en place de l'étape suivante du processus de recherche. On note les réponses de chacune sur un tableau; on note les compétences ou capacités qui reviennent; on additionne les équivalences en termes d'action pour chacune des compétences et des capacités. Enfin nous aboutissons à un classement des compétences que le groupe estime être les plus importantes ou les moins importantes.

our le groupe, cette activité relève d'une technique de résolution créative de problème sous la direction d'un animateur, elle est en fait pour nous, l'étape essentielle de la construction d'un langage commun d'une communauté de pratiques. On retrouve d'ailleurs ici, l'articulation des trois éléments annoncés par Wenger (1998): un engagement mutuel, une entreprise commune, un répertoire partagé.

\section{La troisième fertilisation croisée est celle de l'interaction}

La recherche devient ici, une organisation sociale située co-construite par les chercheurs et les professionnels dans laquelle se déroule un processus d'interaction. En effet, si au 
premier regard, les professionnels peuvent penser que le brainstorming relève d'une technique de coaching, nous y voyons un protocole (de recherche) de production d'informations. Cette production est d'autant plus grande que l'interaction, envisagée comme mise en relation de plusieurs systèmes de pensée est traitée comme un facteur relationnel entre recherche et pratiques. Aboutir à une forme stabilisée de la dyade \{compétence - capacité\} des professionnelles, demande une construction progressive des significations inhérentes à ce type d'échange, mais cette construction est habituellement éludée dans les restitutions des résultats (Bonu, 2004). Ce qui semble normal car les chercheurs ne prennent leurs méthodes comme terrain d'expérience que rarement.

Cette troisième fertilisation est bien la plus féconde car pour agir conjointement avec les participants dès le début de l'action, cela demande une collaboration. C'est-à-dire, convenir ensemble des moyens pour atteindre des buts différents mais non contradictoires (Agostinelli, Riccio, 2010). Convenir ensemble demande a minima de partager un ensemble de règles et de ressources qui facilitent l'interaction; avoir des aptitudes permettant de communiquer efficacement en situation; et avoir une compréhension des échanges à partir d'un langage de sens commun. En d'autres termes, une recherche n'est possible que si les partenaires construisent un point d'interaction entre des communautés de pratique, ici, celle des chercheurs et celle des ChEF.

Cette dimension place de fait la recherche-action participative (Whyte, 1991) dans une démarche stratégique de recherche fondée sur la reconnaissance des discours et des interventions situées. Celle-ci nous pousse à rediscuter non seulement les procédures de recueil de données et les modèles sous-tendus; mais aussi, l'ensemble de règles et de ressources qui permet l'interaction entre des partenaires; et enfin, les modes de communication et leurs caractéristiques d'usage, tels qu'ils sont partagés et compris par ceux qui n'appartiennent pas à la même culture ou communauté. En d'autres termes, il convient de s'intéresser à ce que nous pourrions nommer, la compétence scientifique (je peux faire) : c'est-à-dire, la mise en œuvre des capacités méthodologiques définies comme ce que le chercheur a besoin de savoir pour conduire (je sais faire) effectivement une recherche dans des contextes professionnellement significatifs.

\section{Conclusion}

Lorsqu'on s'interroge sur une possible solution de continuité entre théorie et pratique, l'évidence voudrait que l'on admette la nécessité de penser notre action et d'agir de façon réflexive. La réflexivité nécessaire à la compréhension est alors considérée comme l'expérience discursive d'explicitation de l'implicite, d'expression du tacite qui, lorsque systématisée, caractérise la professionnalité d'une pratique (Couturier, 2002). Dès lors, l'action humaine se réalise sur trois niveaux irréductibles les uns aux autres: le niveau irréfléchi des connaissances tacites et de la conscience pratique; le niveau réfléchi de l'attention et du discours; le niveau réflexif où l'objet de la réflexion est l'expérience personnelle (Eraly, 1994).

Notre première conclusion confirme que quelle que soit la recherche, la pensée et l'action sont étroitement imbriquées. Elle répond directement au premier objectif, celui de démontrer, si cela était encore nécessaire, que la théorie (ou l'académique) et la pratique (ou le terrain) se construisent mutuellement. Ce qui nous conduit à alerter le lecteur sur la confusion entre recherches constructivistes sur des problèmes complexes qui 
demandent une implication du chercheur sur son terrain et une recherche située qui débouche sur une nouvelle posture et une nouvelle inscription du chercheur dans la société.

31 Notre seconde conclusion s'attache particulièrement aux positions et dispositions des acteurs-membres d'un groupe, d'une équipe, dans la mise en scène et en œuvre de leurs connaissances (Meyer, 2008). Pour l'essentiel, les acteurs de terrain sont les savants de leur réalité professionnelle (Boumard, 1989) et les discours sont les significations qu'ils produisent pour stabiliser leurs connaissances professionnelles. Ils prolongent ainsi, des chaînes et systèmes d'interactions qui les font «tenir ensemble» dans une organisation (Hughes, 1996). Ce qui nous intéresse ici, c'est le discours qui explique ou justifie certaines pratiques professionnelles en situation, là où les acteurs produisent des «ce qu'il faudrait faire ", de l'expertise sur la façon dont devraient se comporter les autres dans ce qui touche à leur travail.

Toutefois, si les aspects projectif et participatif ont un intérêt, ils invitent à la prudence des interprétations. L'aspect projectif du discours, étudié à travers le paradigme constructiviste, est considéré comme une totalité de sens. Les significations ainsi énoncées prennent le risque d'un amalgame avec les références à la situation implicite contenue dans la rationalisation. Dès lors, observer les connaissances professionnelles ne serait qu'un exercice d'interprétation. L'aspect participatif observé à partir d'une recherche-action participative (Whyte, 1991) semble être efficace si la démarche contextualise le discours et définit comment celui-ci relie la pratique et la théorie. Il s'agit, en réalité, de redéfinir le paradigme constructiviste en ne négligeant pas les aspects de contextualisation. Le discours n'est pas le reflet du monde extérieur, ni la projection sur le monde extérieur de structures de l'abstraction. Si les conditions énoncées sont réunies, nous pouvons penser alors qu'il n'y a pas solution de continuité entre la théorie et la pratique. Elles sont bien, sur le même continuum.

\section{BIBLIOGRAPHIE}

Agostinelli S. et Riccio P.-M., (2010), Le partenariat entreprise-laboratoire peut-il constituer une communauté de pratiques ?, in Akam N., Lishou C. et Vieira L. (éds.), Le numérique au cœur des partenariats, Dakar, Presses Universitaires de Dakar, pp. 175-186.

Arvanitakis S., Ouvrard M. et Agostinelli S., (à paraître), Partage de compétences dans un réseau d'acteurs, in Riccio P.-M. et Bonnet D. (éds.), Impact des réseaux numériques dans les organisations?, Paris, Presses des Mines, ParisTech.

Albaladejo C. et Casabianca F., (1997), La recherche-action, ambitions, pratiques, débats, Versailles, Quae, 212 p.

Anderson J., (1983), The architecture of cognition, Cambridge, MA USA Harvard University Press, $178 \mathrm{p}$.

Bonu B., (2004), Procédures d'objectivation dans un entretien de recherche, in @ctivités, vol. 1, n², pp. 86-102.

Études de communication, 40 | 2013 
Boumard P., (1989), Les savants de l'intérieur, l'analyse de la société scolaire par ses acteurs, Paris, Colin, $160 \mathrm{p}$.

Callon M. et Latour B., (2006), Sociologie de la traduction : textes fondateurs, Paris, Presses de Mines, $303 \mathrm{p}$.

Coulon A., (2002), L'ethnométhodologie, Paris, PUF, coll. Que sais-je ?, 127 p.

Couturier Y., (2002), Les réflexivités de l'oeuvre théorique de Bourdieu, entre méthode et théorie de la pratique, in Esprit critique, vol. 4, $\mathrm{n}^{\circ}$ 3, www.espritcritique.fr/0403/article2.html, date de la dernière visite $: 19$ décembre 2012.

Eraly A., (1994), L'usage de la psychologie dans le management, l'inflation de la réflexivité professionnelle , in Bouilloud J.-P. et Lécuyer B. (éds.), L'invention de la gestion. Histoire et pratique, Paris, L'Harmattan, pp. 135-159.

Garfinkel H., (1967), Studies in ethnomethodology, Cambridge, Polity Press, 304 p.

Ghiglione R., Kekenbosch C. et Landré A., (1995), L'analyse cognitivo-discursive, Grenoble, Presses Universitaires de Grenoble, $142 \mathrm{p}$.

Ghiglione R. et Blanchet A., (1991), Analyse de contenu et contenus d'analyses, Paris, Dunod, 151 p.

Huberman M. et Miles M., (2003), Analyse des données qualitatives, Bruxelles, De Boeck Supérieur, $632 \mathrm{p}$.

Hugon M.-A. et Seibel C., (1988), Recherches impliquées, recherches-action, le cas de l'éducation, Bruxelles, De Boeck, 185 p.

Hughes E. C., (1996), Le regard sociologique, Essais choisis, Paris, École des hautes études en sciences sociales, $346 \mathrm{p}$.

Kuhn T. S., (1972), La structure des révolutions scientifiques, Paris, Flammarion, $284 \mathrm{p}$.

Landrieux-Kartochian S., (2010), Théorie des organisations, Paris, Gualino, 159 p.

Leplat J., (1991), Compétence et ergonomie, in Amalberti R., de Montmollin M. et Theureau J. (éds.), Modèles en analyse du travail, Liège, Pierre Mardaga, pp. 263-278.

Meyer V., (2008), La méthode des scénarios, un outil d'analyse et d'expertise des formes de communication dans les organisations, in Études de communication, nº 31, pp. 133-156.

Muglioni M., (2008), Emmanuel Kant, Théorie et pratique, Paris, Hatier, 78 p.

Nedeljkovic M., (1975), David Hume, approche phénoménologique de l'action et théorie linguistique du temps, Le Havre, Publication Univ Rouen Havre, 220 p.

Rouleau L., (2007), Théories des organisations, approches classiques, contemporaines et de l'avant-garde, Québec, Presses de l'Université du Québec, 263 p.

Samurçay R. et Pastré P., (1995), La conceptualisation des situations de travail dans la formation des compétences, in Éducation Permanente, $\mathrm{n}^{\circ}$ 123, pp. 13-31.

Suchman L., (1987), Plans and Situated Actions, the problem of human-machine communication, Cambridge, MA USA, Cambridge University Press, 203 p.

Wenger E., (1998), Communities of Practice, Learning, Meaning, and Identity, Cambridge, Cambridge University Press, $318 \mathrm{p}$.

Whyte W. F., (1999), Participatory action research, Newbury Park, Sage Publications, 247 p. 


\section{NOTES}

1. Cette recherche a vu le jour à l'initiative de Mme Faravel, responsable de la commission interne « Entreprenariat au féminin » http://www.cgpme13.fr/mod_inter.php?id=17\&mn_op=4, consulté le 17 janvier 2013.

2. https://www.adum.fr/as/ed/CDAMU/page.pl?page=clubEts, consulté le 17 janvier 2013.

3. Tropes (v8) : www.tropes.fr, consulté le 17 janvier 2013.

\section{RÉSUMÉS}

Cet article a pour but de montrer qu'il n'y a pas de rupture entre la théorie et les pratiques. Nous définissons la recherche sur le terrain comme démarche d'ingénierie qui a la volonté de contrôler des moyens mis à disposition. La recherche est alors une pratique située qui demande une compréhension mutuelle entre le chercheur et le terrain. Cette compréhension facilite la fertilisation croisée des compétences, la reconnaissance des pratiques (recherche et action) et l'interaction. Nous illustrons nos propos à partir d'une recherche qui a pour finalité la création d'un réseau socio-professionnel relationnel de femmes chefs d'entreprise.

This paper aims to show that there is no discontinuity between theory and practice. We define field work as an engineering approach that has the will to control specific means. The research is then a situated practice that asks for mutual understanding between the researcher and the field. This understanding facilitates the cross-fertilization of skills, the recognition of practices (research and action), and the interaction between the two. We illustrate our argument with research aiming at the creation of a social and professional network of women business managers.

\section{INDEX}

Keywords : situated research, understanding, cognitive and discursive analysis, interaction, skill Mots-clés : recherche située, compréhension, analyse cognitivo-discursive, interaction, compétence

\section{AUTEURS}

\section{SERGE AGOSTINELLI}

LSIS, UMR CNRS 7296 - Aix-Marseille Université

Serge Agostinelli est chercheur au Laboratoire des Sciences de l'Information et des Systèmes, UMR CNRS 7296, Aix-Marseille Université. Ses recherches concernent la compatibilité des modèles STIC et SHS. Il porte un regard particulier sur les relations que construisent les hommes et les technologies dans le développement d'application et de services. En fonction des finalités, il utilise les approches expérimentales issues de la psychologie ou les approches ethnographiques 
pour l'analyse des usages et des pratiques professionnelles. Publications récentes : (2011) Communication, Action, Technologie : quelle évaluation des pratiques? Communication \& Organisation, 38, 17-28 ; (2010) Entre structure et action : la compétence communicative des TIC. Questions Vives. Recherches en éducation, 7, 14, 161-174. Adresse électronique : serge.agostinelli@univ-amu.fr.

\section{MARIELLE METGE}

Université du Sud Toulon Var

Marielle Metge est maître de conférences à l'Université du Sud Toulon, Var. Ses recherches portent sur les communautés interactives en ligne et les cultures communautaires avec un regard particulier sur les situations de communication, d'interactions, d'actions, de sens partagé. La sémiologie et la sémiotique associée à la pragmatique, essentiellement centrée sur la relation et le contexte, permettent d'axer ses recherches à la fois sur l'articulation signifiants-signifiés, mais aussi sur les effets produits en pratique dans le cadre de ces situations communautaires ou de l'usage de produits de communication multimédias en ligne. Publication récente : (2011) Entre communauté de pratique et d'apprentissage : un espace commun de communication des connaissances, Éducation, Science \& Society, (2), 1, 41-53. Adresse électronique : metge@univ-tln.fr. 